\title{
Neonatal Agenesis of Corpus Callosum in a Pregnant Lady with Multiple Endocrine Neoplasia Type I
}

\author{
Dabbour $\mathrm{S}^{1 *}$, Aref $\mathrm{A}^{2}$ and Rengert $\mathrm{E}^{1}$ \\ ${ }^{1}$ Princess Alexandra Hospital, United Kingdom \\ ${ }^{2}$ The Fetal Medicine Unit, University College Hospital London, UK \\ Submission: August 27, 2018; Published: September 19, 2018 \\ *Corresponding author: Sheriff Dabbour, Princess Alexandra Hospital, Hamstel Rd, Harlow CM20 1QX; UK, Email: sheriff.dabbour@nhs.net
}

\begin{abstract}
This is the first reported case of a pregnant woman diagnosed with Multiple Endocrine Neoplasia type 1 (MEN Type I) who delivered a baby with agenesis of the corpus callosum (ACC), colpocephaly and some dysmorphic facial features. (MEN Type I) is a rare inherited disorder caused by tumor of the neuroendocrinal tissue and may be associated with some clinical symptoms. The main aim of management is to assess and manage potential complications that might happen to the mother and her baby [1,2]. The corpus callosum (CC) is the flat bundle of white matter commissural fibers beneath the cerebral cortex spanning part of the longitudinal fissure and connecting the left and right cerebral hemispheres. Agenesis of corpus callosum (ACC) may be associated with early seizures, eye abnormalities, hydrocephalus, developmental delay and other abnormalities. Colpocephaly is a cephalic disorder involving the disproportionate enlargement of the occipital horns of the lateral ventricles and is usually diagnosed by cranial ultrasonography early after birth. We report a case of a 35-year-old multigravida with a confirmed diagnosis of MEN Type I prior to conception. Antenataly, pregnancy was followed up as a high-risk pregnancy with regular fetal growth scans which identified a growth restricted fetus. An emergency Caesarean section was performed at 37 weeks gestation for a fetal growth restriction. The neonate was born in good conditions but had some dysmorphic facial features and jitteriness. Neonatal cranial ultrasonography and brain MRI confirmed ACC and colpocephaly. This case report highlights the importance of comprehensive neonatal clinical examination and imaging in such rare conditions.
\end{abstract}

Keywords: Multiple endocrine neoplasia; Corpus Callosum; Colpocephaly

Abbreviations: ZES: Zollinger-Ellison Syndrome; CC: Corpus Callosum; USS: Ultra Sound Scans; PTH: Parathyroid Hormone; AAM: Alanine Aminotransferase

\section{Introduction}

Multiple endocrine neoplasia type 1 (MEN Type I) is a rare inherited tumor of the neuroendocrinal tissue that is caused by inactivating mutations of the MEN1 tumor suppressor gene at the 11q13 locus with a combination of parathyroid tumors, pancreatic islet cell tumors and anterior pituitary tumors. Clinically, MEN 1 is defined following development of two out of these three tumors. The pattern of inheritance is usually an autosomal dominant pattern, however still sporadic cases have been reported due to new mutations. Most MEN Type I tumors are not aggressive and may remain asymptomatic for years $[1,3]$. On the other hand, patients may present with signs and symptoms of primary hyperparathyroidism which is the most common and the initial manifestation of (MEN1). Other patients may present with Zollinger-Ellison syndrome (ZES) with gastrinoma symptoms caused by peptic ulcers and esophagitis. Other complications like hypercalcaemia and hypoglycemia might be associated. Due to the rare nature of MEN 1, there are no guidelines on how pregnant women should be managed and the management only depends on treating any potential complications that might happen.
The corpus callosum (CC) is that flat bundle of white matter commissural fibers beneath the cerebral cortex spanning a part of the longitudinal fissure and connecting the left and right cerebral hemispheres. Formation of the corpus callosum begins as early as six weeks of gestation, with the first fibers crossing the midline at 11-12 weeks of gestation. The basic shape is completed by 18-20 weeks of gestation. Therefore, a proper prenatal sonographic evaluation can be performed only after 20 weeks. ACC is a rare disorder with reported incidence of $0.05-0.7 \%$. It can be associated with mental retardation, seizures, developmental disabilities, ocular anomalies, gyral abnormalities, hydrocephalus, hyper mobility disorders, central nervous system lesions, costovertebral defects, colpocephelay and increased the risk of early onset seizures.

\section{Case Report}

\section{Background information}

We reported a 35-year-old mutiparous with a known history of MEN Type I. She was diagnosed by detecting the mutation in MEN I tumor suppressor gene at the 11q13 locus following 
genetic family screening because of her family history of MEN type I in her mother. She later developed pituitary micro adenoma, parathyroid and pancreatic masses and had been under regular endocrinology team follow up. Genetic testing had been undertaken for both her previous live birth children, which detected the MEN Type I in her elder daughter. Prior to conception, she had regular endocrinology review to ensure normal serum calcium level before trying to conceive. She has been having regular annual endocrinology team review, annual MRI scans and three yearly cranial ultra sound scans (USS). There had been no changes with the size of the pituitary adenoma or pancreatic masses.

\section{Management during pregnancy}

The 35 year old woman was managed under multidisciplinary specialized obstetric care joint with endocrinology team due to the pituitary micro-adenoma. Atbooking her Body mass index was 25.8, serum Ca was $2.46 \mathrm{mmol} / \mathrm{L}$, serum Phosphate $1.07 \mathrm{mmol} / \mathrm{L}$, Alkaline Phosphatase $93 \mathrm{IU} / \mathrm{L}$, serum sodium (Na) 138mmol/L, serum potassium $(\mathrm{K}) 4.2 \mathrm{mmol} / \mathrm{L}$, serum creatinine $53 \mathrm{umol} / \mathrm{L}$, urea $3.2 \mathrm{mmol} / \mathrm{L}$, Parathyroid Hormone (PTH) 0.6pmol/l and prolactin levels was high $3626 \mathrm{mU} / \mathrm{L}$. The 11-14 week combined screening test showed an increased risk of trisomies 1:91 (Cutoff risk used 1:150). Non Invasive prenatal testing and chorionic villous sampling was undertaken and excluded Trisomy 13,18 and 21 . At 20 weeks gestation, the routine anomaly ultra sound scan was undertaken and it showed normal fetal anatomy. Second trimester fetal echocardiography revealed normal fetal cardiac structural anatomy. At 25 weeks gestation the woman was suspected of having obstetric cholestasis due to raised alanine aminotransferase (ALT) levels and excessive itching.she was managed conservatively, with excessive fetal monitoring, weekly serum Bile acids and close monitoring of serum calcium.

Regular fetal growth scan was conducted from 28 week. At 35 weeks gestation fetal growth restriction was diagnosed and an elective caesarean section was planned between 37-38 weeks with neonatal team input. At $37+2$ gestation the woman experienced spontaneous rupture of membranes (SROM) and an emergency caesarean section was performed due to intrapartum abnormal fetal heart rate pattern.

\section{Neonatal management}

A baby girl was delivered at 37 weeks +2 days gestational age. She cried immediately after birth with Apgar score of 7 and 9 at 1 and 5 minutes respectively. The birth weight was $2.1 \mathrm{Kg}$. The baby has symmetrical intrauterine growth restriction, both birth weight and head circumference on the growth chart were under the 0.4 th centile. On the initial examination, the baby was jittery and has dysmorphic features in the form of microcephaly, micrognathia, short, narrow and upslanting fissures, no spontaneous eye opening, possible sub mucus cleft palate, hypertolerism, small upward slanting eyes, prominent nasal bridge, small mouth, receding shin, hole at the front of the upper gum, bilateral single palmar creases, fifth finger clinodactyly and prominent clitoris with tags. The baby's tone was slightly reduced. Otherwise, the rest of the clinical examination was normal including heart, chest, abdominal and limb examination (Figure 1). The baby has had a nasogastric tube in place for nearly a week after that she managed to have feeds through the bottle.

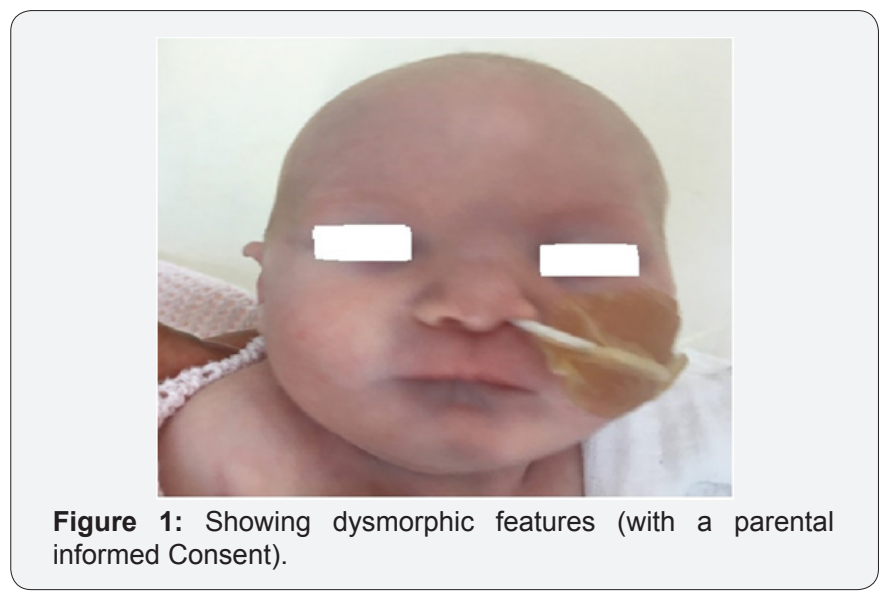

On day 2, cranial ultrasound showed absent corpus callosum and dilated posterior part of the lateral ventricles. And day 3 blood tests (renal functions, liver functions, serum electrolytes, bone profile, serum cortisol, serum ACTH, serum TSH and Free T4) were all normal (Figures 2-5). Blood sugar was $3.4 \mathrm{mmol} / \mathrm{L}$, serum Ca 2.56mmol/L, serum Ph 2.02mmol/L, ALP $122 \mathrm{IU} / \mathrm{L}$; Cortisol $139 \mathrm{nmol} / \mathrm{L}$. TORCH screen was negative. Genetic tests and microarray were sent to screen for MEN and other genetic syndrome and mutations. On day 4, brain MRI confirmed the absence of corpus callosum and colpocephaly with normal MRI. The initial newborn hearing screening test revealed bilateral moderate hearing loss. The basic chromosomal test was normal, but further extended genetic tests and microarray were sent to screen for MEN gene mutations and other genetic syndrome and mutations.

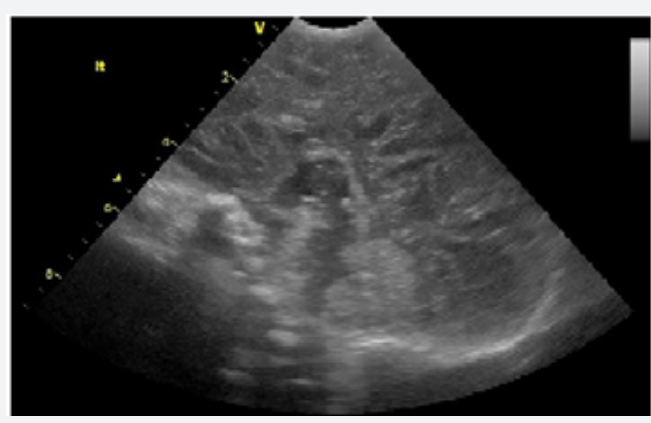

Figure 2: Cranial ultrasound with a sagittal view of the brain showing absent corpus Callosum.

The baby had a small mouth and suspected sub mucosal cleft palate. The baby was managed with involvement of the cleft palate team. Feeding was started via bottle and toping up using nasogastric tube. The baby was well tolerating formula feeding. A multidisciplinary discharge meeting was done at Day 6 before the discharge. Recommendations for genetic, hearing, ophthalmology, physiotherapy, community, local consultant clinic follow up, and cleft palate team. 


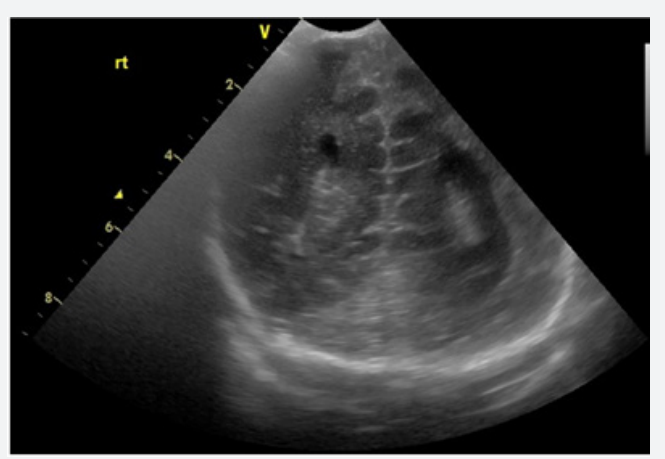

Figure 3: Cranial Ultrasound showing a coronal Brain view with evident colpocephaly and dilated lateral ventricle.

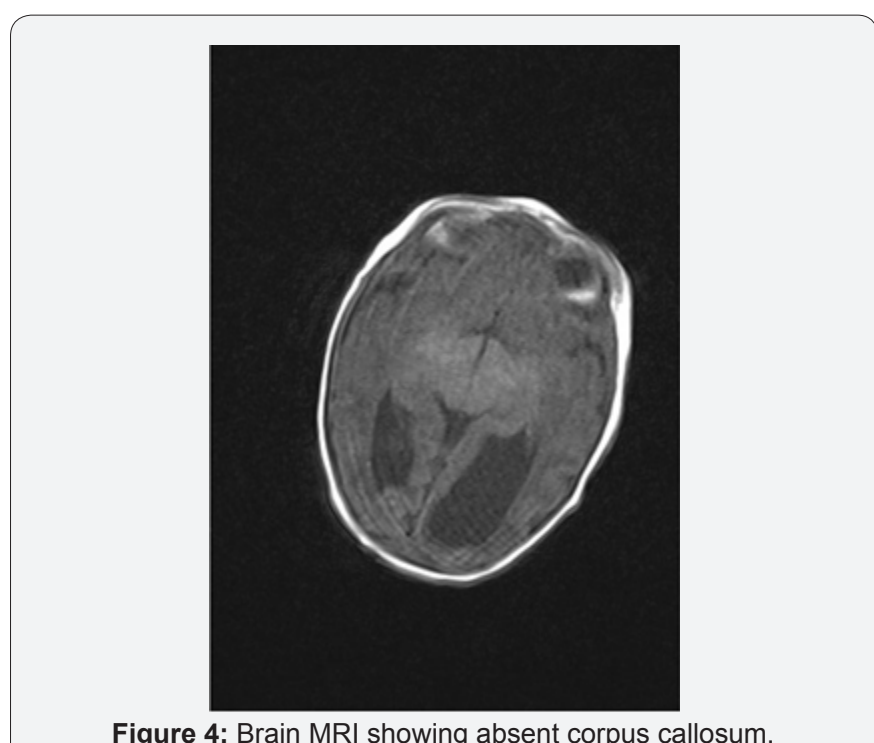

Figure 4: Brain MRI showing absent corpus callosum.

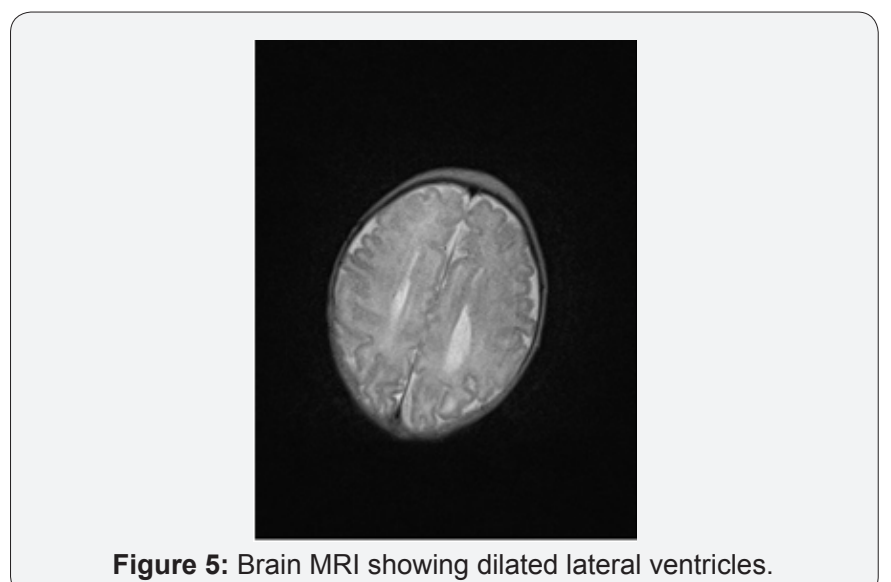

Discussion

Multiple endocrine neoplasia Type I (MEN 1) is usually inherited as an autosomal dominant disorder, but still sporadic cases occur due to new mutations. The reported prevalence is rare and about $0.25 \%$. Thus there is no clinical guidance of management of this condition during pregnancy, due to the rare reported cases of pregnancies associated with MEN.
Management of these pregnancies is challenging, and depends on the controlling the presenting symptoms and monitoring maternal and fetal well being. Pregnant women with hyperparathyroidism are usually liable to complications especially urinary tract stones, hyper emesis gravidarum, maternal arrythmis secondary to hypercalcaemia.

Regular antenatal multidisciplinary care is essential to prevent and early manage maternal and fetal complications like the increased risk of gestational hypertension, pre-eclampsia, Fetal Growth Restriction, and preterm delivery. In uncontrolled maternal hypercalcaemia, the neonate is at risk of persistent hypocalcaemia due to permanent neonatal hypoparathyroidism in the fetus that develop secondary to failure of the fetal parathyroid gland development and may develop seizures following birth.

Managementisusually conservativewithin a multidisciplinary team to target a normo-calcaemic diet, adequate hydration and regular monitoring of maternal blood tests (serum urea, electrolytes, total serum and corrected calcium and serum phosphate levels) and regular fetal growth and well being scans aiming to achieve a good pregnancy outcome.

Agenesis of the corpus callosum (ACC) is a developmental disorder that can occur in isolation or in association with other central nervous system (CNS) or systemic malformations. ACC can be partial or complete absence of the main commissural pathway that connects the two cerebral hemispheres. The corpus callosum (CC) can be assessed on ultrasound by direct visualization, or by presence of indirect features, such as ventriculomegaly, absence of the cavum septi pellucidi or widening of interhemispheric fissure.

ACC has been associated with several syndromes with autosomal dominant, autosomal recessive or X-linked mode of inheritance. The more common genetic causes of callosal agenesis are; Aicardi syndrome: found almost exclusively in girls as it is inherited as an X-linked dominant trait; it is characterized by the presence of ACC associated with chorioretinal abnormalities, infantile seizures and mental retardation. Also ACC can be present in Andermann syndrome: a rare autosomal recessive disorder characterized by ACC, progressive motor-sensory neuropathy and mental retardation. Hallermann-Streiff syndrome is another rare congenital condition characterized mainly by abnormalities of the skull and facial bones, characteristic facial features, sparse hair, eye abnormalities, dental defects, degenerative skin changes and proportionate short stature. Intellectual disability is present in some individuals. Almost all reported cases of this condition appear to have occurred sporadically and are thought to have resulted from a new mutation in the affected individual. LujanFryns syndrome is also another rare condition characterized by intellectual disability, behavioral problems, and poor muscle tone (hypotonia), a large head (macrocephaly); and a thin face with distinctive facial features (prominent top of the nose, narrow roof of the mouth, crowded teeth and a small chin). Most 
of the cases occur in males. Lujan-Fryns syndrome is caused by mutations in the MED12 gene which is so close to the gene locus responsible for MEN.

Due to the rarity of ACC, it is very difficult to establish the frequency of underlying genetic syndromes. Bedeschi et al. studied 63 cases of ACC with a neuropsychiatric disorder (mental retardation of varying severity, learning disabilities or epilepsy) and found that $33 \%$ of the cases had a recognizable syndrome [4]. Schell-Apacik studied 41 patients with ACC and found that $12 \%$ had a genetic syndrome [5]. Due to the extreme rarity of the condition, the baby was recruited into the UK 100,000 genomic project. This extremely rare case reports highlights the importance of multidisciplinary care for pregnant women with rare medical disorders during the antenatal period.

\section{References}

1. Daglar HK, Kirbas A, Biberoglu E, Laleli B, Danisman N, et al. (2016) Management of a multiple endocrine neoplasia type 1 during pregnancy: A case report and review of the literature. J Exp Ther Oncol 11(3): 217-220.

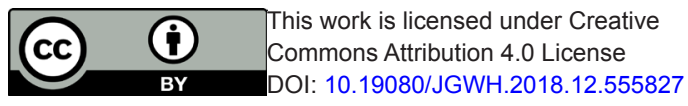

2. Mistry M, Gupta M, Kaler M (2014) Pregnancy in multiple endocrine neoplasia type 1 equals multiple complications. Obstet Med 7(3): 123125 .

3. Donckier JE, Michel L (2012) Multiple endocrine neoplasia 2A and pregnancy: a medical and ethical challenge. Acta Clin Belg 67(1): 5455.

4. Bedeschi MF, Bonaglia MC, Grasso R, Pellegri A, Garghentino RR, et al. (2006) Agenesis of the corpus callosum: clinical and genetic study in 63 young patients. Pediatr Neurol 34(3): 186-193.

5. Schell-Apacik CC, Wagner K, Bihler M, Ertl-Wagner B, Heinrich U, et al. (2008) Agenesis and dysgenesis of the corpus callosum: clinical, genetic and neuroimaging findings in a series of 41 patients. Am J Med Genet A 146A(19): 2501-2511.

Your next submission with Juniper Publishers will reach you the below assets

- Quality Editorial service

- Swift Peer Review

- Reprints availability

- E-prints Service

- Manuscript Podcast for convenient understanding

- Global attainment for your research

- Manuscript accessibility in different formats ( Pdf, E-pub, Full Text, Audio)

- Unceasing customer service

Track the below URL for one-step submission https://juniperpublishers.com/online-submission.php 